\title{
Screening of Damage Regions in Retinopathy Using Segmentation-Color Space Selection
}

\author{
Jyoti Patil, Sharmila Chaudhari \\ Baburaoji Gholap A.S.C. College, Pune, India
}

\begin{abstract}
Screening is essential for extraction of object Candidate in Diabetic Retinopathy (DR).Purpose is to detect aberrant from color fundus images. Pixels with number of lesions can be illuminated by region Segmentation Using color space selection. In the proposed method simple color detection in $L A B$ color space has done. Four color models, like RGB, $L u v, L a b$ and HVC, are calculated which uses the object-based color difference image. The objectbased color difference images of bright and dark objects are detected respectively. This helps in objects detection in Diabetic retinopathy like exudates hemorrhages and blood vessel. Thus by computing the color difference we can easily classify objects. Such a screening systems may reduce efforts of ophthalmologist. In this paper our goal is to analyze and evaluate the various color spaces in color image enhancement applications. Conversion accuracy and similarity measure are the two objective parameters to measure the performance of each color space [1].
\end{abstract}

\section{Introduction}

Diabetic Retinopathy (DR) is a major disease amongst the eye diseases in the retina. Its features are Hemorrhages (H), Micro aneurysms (MA), and Exudates (hard exudates (HE) and soft exudates (SEx). For the early detection of DR, ophthalmologist need to examine the fundus structure of posterior pole of the eye that includes the retina, macula, fovea, optic nerve, OD, blood vessels and other interior structures. We have use simple color detection in LAB color space selection. The RGB image is converted to LAB color space and then the user draws some freehand-drawn irregularly shaped region to identify a color. The color difference in LAB color space is then calculated for every pixel in the image between that pixel's color and the average $\mathrm{LAB}$ color of the drawn region. This classifies and segments retinal images. Another problem is that even under equal intensity, some colors are visually brighter than others. Different color representations try to overcome these problems, with varying degrees of success [1]. It is for this reason that there are so many standard color representations. Eight most common color spaces are formulated and tested in this paper. Their conversion efficiency is computed and they are evaluated based on their performance in image enhancement applicability [2]. A new image contrast enhancement method based on ulti-solution decomposition is proposed and tested for all the color spaces [1].

\section{Literature Review}

Rmasubramanian stated that the image is preprocessed and segmented using CIE Lab color space. The segmented image along with Optic Disc (OD) is chosen [5]. By assessing the algorithm's performance while conducting experiments with the colour space selection, both luminance and colour correction, and background class information, the use of colour in the detection of diabetic retinopathy was quantitatively evaluated by Kauppi et al. [6].

\subsection{Proposed Method}

In Proposed methods is enhancement method is use for Classification and segmentation of $\mathrm{HE}$, screening of Hemorrhages, EXs, the OD, and blood vessels. Accurate segmentation of EXs based on a chosen classification scheme.

\subsection{Color Space Selection}

To increase the Contrast HEs and the background such as histogram based image normalization and contrast enhancement method is applied. When color spaces are used it is extracting the dominant retinal features in an optimum way than the original RGB color space is. For this a set of color spaces such as RGB, HSL, Lab, LUV, CMYK were tested and compared for their efficacy in robust and useful extraction of features. Color space selection The first concern with tri chromatic photometric information is the color space selection. There are numerous color spaces available, but the appropriate color space is typically selected for each application separately since none of the color spaces is universally better than the others. However, it is assumed that any particular space constructed using a linear transformation from another should not affect the results. Any linear color space from the RGB values is given by 


$=\quad \begin{array}{ll}h 11 & h 12 \\ h 21 & h 22 \\ h 31 & h 32\end{array} *$

where the $3 \times 3$ matrix defines a linear transformation to the color space RGB.

The search for suitable colour space is further restricted to two issues that can affect the analysis: expert annotations and lesion separability in the colour space. Since the expert annotations have been performed manually by medical doctors using RGB displays, it is motivating to study whether the colour representation has any effect on the analysis. If it had, colour spaces designed to model the human colour vision (such as CIE L*a*b*) would represent the ground truth better. Another important issue that directly contributes to the ability of the baseline algorithm to discriminate the lesions is how photometric cues of lesions are distributed in the colour space. To study the addressed issues, two delicately selected colour spaces are used in the experiments: CIE L*a*b* and HSV. Instead of experimenting with all the possible alternatives, the selected colour spaces are used to indicate if further experiments are required. 5.4.1 CIE L*a*b* CIE $\mathrm{L}^{*} \mathrm{a} \mathrm{b}^{*}$ is a perceptually uniform color space recommended by the Commission International d'Eclairage (CIE) [27], where $L^{*}$ denotes the lightness axis and the color opponents $a^{*}$ and $b^{*}$ define the red-green and yellow-blue axes. In theory, a unit Euclidian distance in CIE $\mathrm{L}^{*} \mathrm{a} * \mathrm{~b} *$ is the threshold for a human to distinguish color difference. The colour transformation from XYZ to CIE L*a*b* color space is a non-linear mapping. Photometric cue in lesion detection and defined as

$$
\begin{aligned}
& \mathrm{L}^{*}=116 f\left(\frac{Y}{Y N}\right)-16, \\
& \mathrm{a}^{*}=500\left[f\left(\frac{X}{X N}\right)-f\left(\frac{Y}{Y N}\right)\right], \\
& \mathrm{b}^{*}=200\left[f\left(\frac{Y}{Y N}\right)-f\left(\frac{Z}{Z N}\right)\right],
\end{aligned}
$$

where

$$
f(x)=\left\{\begin{array}{r}
t \frac{1}{3}, t>0.008856 \\
7.787 t+\frac{16}{116},
\end{array}\right.
$$

otherwise Here $\mathrm{X}, \mathrm{Y}$ and $\mathrm{Z}$ are the red, green and blue for standard observer (CIE $1931 \mathrm{XYZ}$ ), and $\mathrm{XN}, \mathrm{YN}$ and $\mathrm{ZN}$ define the illuminant dependent reference white point in the XYZ color space. In general, the characteristics of the illuminant should be known when transforming RGB to XYZ values, and further to the $\mathrm{L}^{*} \mathrm{a} \mathrm{b}^{*}$ color space. However, if image capturing is conducted using the same camera under the same illuminant then the white point in the transformation can be fixed, e.g. CIE standard illuminant D65 [5].
Two color spaces, which have enhanced contrast and uniformity for EXs and other retinal structures, were selected for further analysis. These were HSL (hue, saturation and lightness/value/intensity), and GLM' (G-component from RGB, L-component from LUV. RGB (Red, Green, Blue) RGB is most common being used in virtually every computer system, television and video. It is easy to implement. However, high correlation between channels, significant perceptual non-linearity with visual perception, device dependency and mixing of chrominance and luminance data make RGB not a very favorable choice for color image processing. Thus we seek for another color spaces which are more suitable for processing [5].

\subsection{HSV/HSI (Hue Saturation Lightness Value/ Intensity)}

This is a non-linear transform of the RGB color space. Separation of the luminance component from the chrominance information makes it advantageous in image processing. It is also extremely intuitive. However, it is non linear and device dependent. It is widely used in the field of color vision.

In a typical retinal image, the red component is over saturated and has low contrast over the bright regions. The blue component is under saturated and has low contrast over the black regions. Only green part has enhanced contrast over the whole region. This makes the image to have very low contrast which is signified by the grouping of large peaks in a small area on the histogram plot. The first color space was selected since it is believed to have similarity with human color perception and it is less affected by inter and intra image variations due to various artifacts, hence is potentially helpful for our abnormality (see Figure 1 - Sample original image with three color components (a), red component (b), green component (c), and blue component (d)).

The original RGB image was converted to HSL color using the following formula [1]:

$$
\begin{aligned}
& \mathrm{H}=\arctan \left(\frac{V_{2}}{V_{1}}\right), \mathrm{S}=\sqrt{v 1^{2}+v 2^{2}}, \mathrm{~L}=\left(\frac{R+G+B}{\sqrt{3}}\right) \\
& \mathrm{V} 1=\left(\frac{2 R-G-E}{\sqrt{G}}\right) \text { and } \mathrm{v} 2=\left(\frac{R-G}{\sqrt{2}}\right)
\end{aligned}
$$

Now, the illuminate corrected image from for Eq. 5.11 can be recovered from the fitted result as follows:

$$
\mathrm{I}_{0}(\mathrm{x}, \mathrm{y})=\frac{\mathrm{I}(\mathrm{x}, \mathrm{y})}{\mathrm{t}(\mathrm{x}, \mathrm{y})}
$$

is the fitted parametric model value at image location is the fitted parametric model value at image location (x, y). In the RGB color space the natural choice for 
the background point extraction and the image luminance estimation is the intensity $[\mathrm{R}+\mathrm{G}+\mathrm{B}] / 3$, but in the case of eye fundus images the red channel can be used directly [138]. The distortion free RGB image can be recovered simply by dividing pixelwise each color channel of the image separately with the same correction factor as follows,

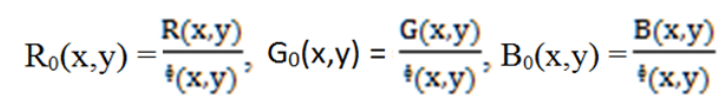

are the color channels of the luminance corrected image, and the corresponding channels of the luminance degraded image [5].

\subsection{GLM' Color Bands for Enhancing Features of DR}

The green channel of the original color fundus image is used for study DRs. Intensity information can be gotten from algorithms for color images. The green channel of fundus retinal images shows higher contrast for HEs, So we use HE for detection and segmentation. Three different channels has been combined to detect aberration in DR and OD localization in fundus images. The G-channel from RGB color space is use for fundus images, so we can observe HEs appear brighter than the background, taken up by the green channel. The L channel in the LUV color space was selected as a second channel, since luminosity information has uniformity for EXs and OD [1], [2]. In most fundus images HEs appear yellowish, while background color is red. We use third channel from color space as it gives great separation with dark red blood vessels and OD.

Table 1. Resultant values of Color space selection Images

\begin{tabular}{|c|c|c|c|}
\hline Figure & $\begin{array}{l}\text { The Mean LAB } \\
\text { for RGB colors }\end{array}$ & $\begin{array}{l}\text { The Mean } \\
\text { Delta E in } \\
\text { Mask region }\end{array}$ & $\begin{array}{l}\text { The Average } \\
\text { color in the } \\
\text { region drawn }\end{array}$ \\
\hline Fig 1 & $85.5,9.72,53.98$ & $\begin{array}{l}20.58+/ \\
11.40\end{array}$ & 54.8 \\
\hline Fig 2 & $47.80,34.27,55.52$ & $\begin{array}{l}20.08+ \\
13.14\end{array}$ & 59.5 \\
\hline Fig 3 & $64.20,26.6,21.94$ & $\begin{array}{l}22.69+\quad /- \\
13.15\end{array}$ & 62.1 \\
\hline Fig 4 & $62.91,27.05,68.43$ & $\begin{array}{l}11.06+ \\
10.82\end{array}$ & 43.5 \\
\hline
\end{tabular}

\section{Result and Discussion}

Segmented exudates: In Figure 1 we have done accurate segmentation of positive optic disc region is been separated from the candidate. Also we have done segmentation of blood vessels. At the same segmentation of yellowish color hard exudates.

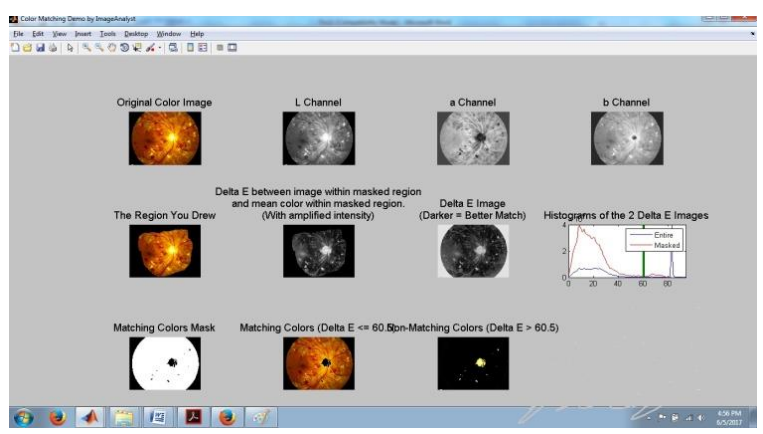

Figure 1. Optic disc and Blood vessels segmentation

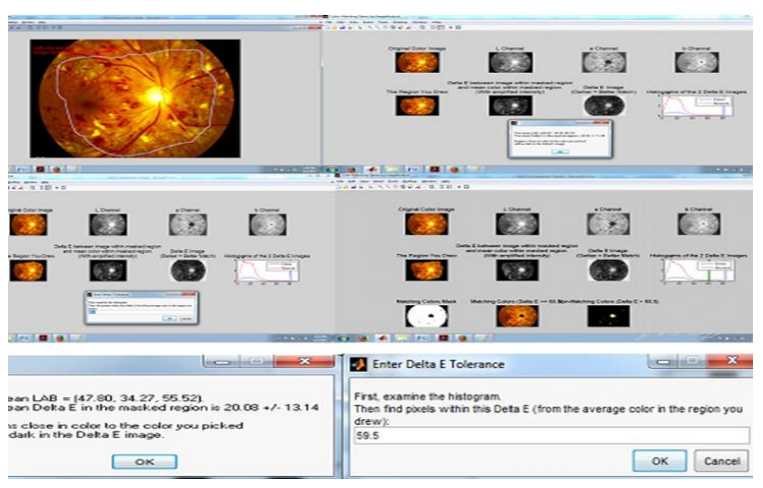

Figure 2. Optic disc and Blood vessels segmentation

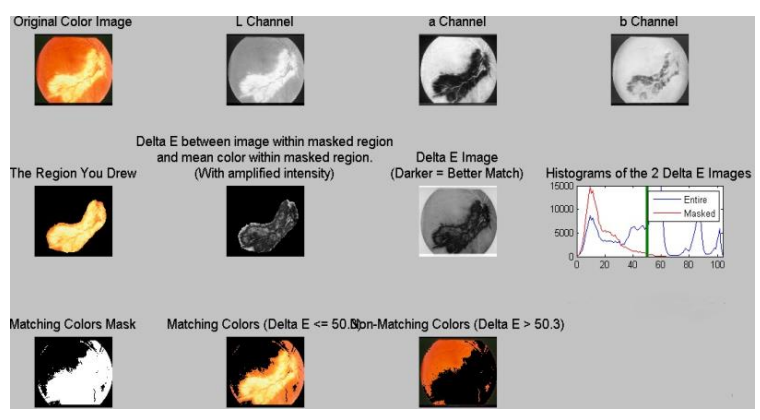

Figure 3. Optic disc and Blood vessels segmentation

In Figure 4, We have selected damage area which is giving segmented region with amplified Intensity. An active contour-based technique was developed to produce a more accurate boundary. The Damaged region is invariably fragmented into multiple regions by blood vessels. In L Channel, a Channel and b Channel, we can observe $1 \mathrm{D}$ vector of only the pixels within the masked area, from Get Mean LAB Values.

Also plotted the histograms of the pixels in both the masked region and the entire image.

In Figure 5, we have selected cotton wool spot in Diabetic retinopathy. Thus, we used a color morphology preprocessing smoothing to remove the vessels and create a fairly constant region before applying a snake method. Here we implemented the concept of color morphology in different color 
spaces including HLS, LCh, and Lab. We also demonstrated the improvement obtained using the proposed color morphological closing over graylevel morphology for this application.

In Figure 6, we have segmented hemorrhages region showing yellowish damages. We can Cleary observe Damaged region because background is been removed from front ground. Algorithm has generated histogram.

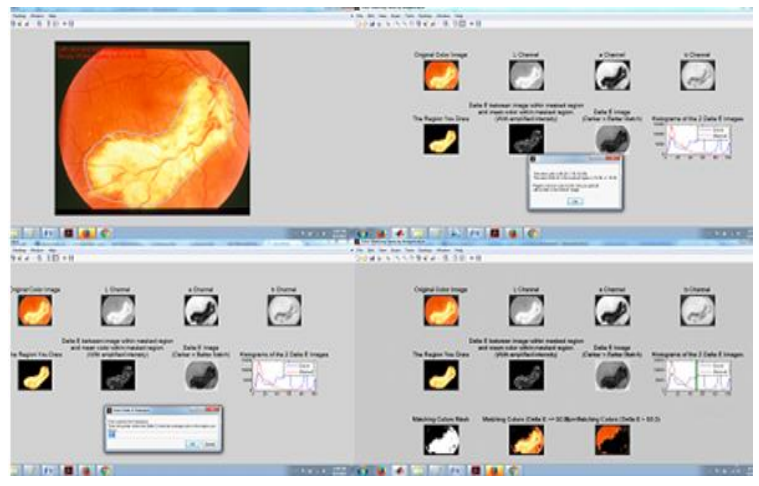

Figure 4. Selected Damaged area with Damaged vessels

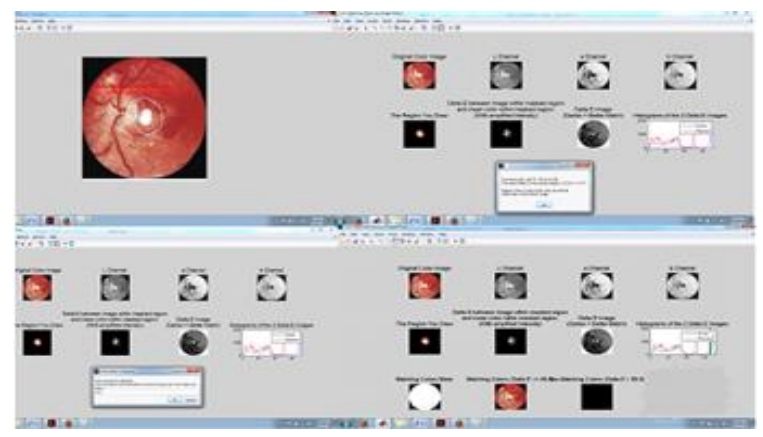

Figure 5. Selected Cotton wool spot

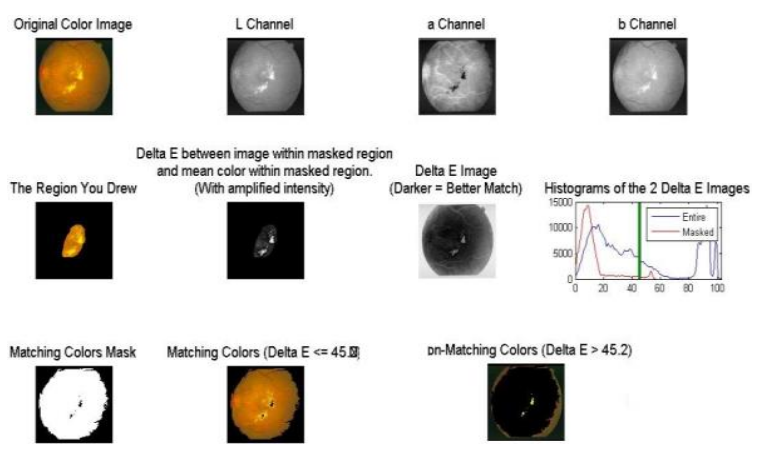

Figure 6. Segmented Hemorrhages Region

\section{Conclusion}

We applied different threshold values which are the limit boundary of the best exudates color similarity which can segmentation process according to the color. The value of $\mathrm{T}$ can be customized to the preferred level of precession similarity. The higher the threshold value used the harder the exudates, otherwise decrease the value of $\mathrm{T}$ for soft exudates (e.g., cotton wool spot). The obtained accuracy value for exudates detection using color references space I $\mathrm{s} 95.54 \%$ using ground truth data of image comparison according to the color of the exudates area. We have segmented hemorrhages region showing yellowish damages. We can Cleary observe Damaged region because background is been removed from front ground. Algorithm has generated histogram. we have selected cotton wool spot in Diabetic retinopathy. Thus, we used a color morphology preprocessing smoothing to remove the vessels and create a fairly constant region before applying a snake method. Here we implemented the concept of color morphology in different color spaces including HLS, LCh, and Lab. We also demonstrated the improvement obtained using the proposed color morphological closing over graylevel morphology for this application. We have segmented hemorrhages region showing yellowish damages. We can Cleary observe Damaged region because background is been removed from front ground. Algorithm has generated histogram.

\section{References}

[1]https://in.mathworks.com/matlabcentral/fileexchange/31 118-color-segmentation-by-delta-e-color-difference. [Access Date: 26 September, 2016].

[2] An Automated Segmentation of Retinal Images for use in Diabetic Retinopathy Studies By Daniel Moges Tadesse, Addis Ababa University.

[3] Jelinek, H., Cree, M., (2010). Automated Image Detection of Retinal Pathology. Boca Raton: CRC Press.

[4] Patil, J., (2012). Development of Digital Image Processing tool. LAMBERT Publication, Germany.

[5] Ramasubramanian, B., Prabhakar, G., (2013), An Early Screening System for the Detection of Diabetic Retinopathy using Image Processing, International Journal of Computer Applications, Volume 61 - No.15, January.

[6] Kauppi, T., Kalesnykiene, V., Kamarainen, J.-K., Lensu, L., Sorri, I., Uusitalo, H., Kälviäinen, H., and Pietilä, J., (2006). DIARETDB0: Evaluation database and methodology for diabetic retinopathy algorithms. Published online at: http://www2.it.lut.fi/project/imageret/ diaretdb0/. 